\title{
Long-Baseline Oscillation Experiments as a Tool to Probe High Energy Flavor Symmetry Models
}

\author{
Pedro Pasquini (iD) ${ }^{1,2}$ \\ ${ }^{1}$ Instituto de Física Gleb Wataghin-UNICAMP, 13083-859 Campinas, SP, Brazil \\ ${ }^{2}$ Northwestern University, Department of Physics \& Astronomy, 2145 Sheridan Road, Evanston, IL 60208, USA \\ Correspondence should be addressed to Pedro Pasquini; pedrosimpas@gmail.com
}

Received 2 February 2018; Revised 19 March 2018; Accepted 22 March 2018; Published 4 July 2018

Academic Editor: Giorgio Arcadi

Copyright (c) 2018 Pedro Pasquini. This is an open access article distributed under the Creative Commons Attribution License, which permits unrestricted use, distribution, and reproduction in any medium, provided the original work is properly cited. The publication of this article was funded by $\mathrm{SCOAP}^{3}$.

\begin{abstract}
We review the current status of neutrino oscillation experiments, mainly focusing on $\mathrm{T} 2(\mathrm{H}) \mathrm{K}, \mathrm{NO} v \mathrm{~A}$, and DUNE. Their capability to probe high energy physics is found in the precision measurement of the CP phase and $\theta_{23}$. In general, neutrino mass models predict correlations among the mixing angles that can be used to scan and shrink their parameter space. We updated previous analysis and presented a list of models that contain such structure.
\end{abstract}

\section{Introduction}

The upcoming sets of long-baseline neutrino experiments will establish a new standard in the search for new physics. Two distinct directions arise; the phenomenological approach consists of seeking new unobserved phenomena that are present in a large class of models. They were extensively studied in the literature and are subdivided into 3 main groups: Nonstandard Interactions (NSI) searches [114], Light Sterile Neutrinos [15-19], and Nonunitarity [2028]. The second approach is more theory based and was less explored. It focuses on correlations among neutrino mixing angles predicted by high energy models. This opens the possibility of testing models that contain almost no lowenergy phenomenological effects different from the Standard Model.

Since the discovery of neutrino oscillations, a plethora of models was realized to try to explain the origin of the neutrino masses. The first proposal was the see-saw mechanism [29-34] which tried to explain the smallness of neutrino masses $\left(m_{\nu}\right)$ through a heavy mass scale $(M) m_{\nu} \propto$ $M^{-1}$. Another possible path uses loop mechanisms, in which neutrino masses can be suppressed at zeroth [35] or even first order [36]. Nevertheless, such theories usually do not explain the structure of the oscillation parameters, as they are merely free parameters.
This changes by the addition of discrete symmetry that controls the pattern of the leptonic mass matrix [37-39]; for a review on the subject see, e.g., $[40,41]$. They can predict relations among the neutrino mixing angles [42-53] which can be used to constrain the parameter space of such theories [54].

This manuscript is divided into seven sections: In Section 2 we describe current and future neutrino oscillation experiments: $\mathrm{T} 2 \mathrm{~K}, \mathrm{NO} v \mathrm{~A}$, and DUNE and their simulation. In Section 3 we briefly discuss the statistical analysis and methods used to scan the parameter space. In Section 4 we present the sensitivity to neutrino mixing parameters expected in each experiment. In Section 5 we review the possibilities of using the $\theta_{23}-\delta_{\mathrm{CP}}$ correlation in long-baseline experiments by updating previous analysis of two models $[55,56]$. In Section 6 we review the possibility of using the $\theta_{13}-\theta_{23}$ correlation by combining long-baseline experiments with reaction measurements of $\theta_{13}$. In Section 8 we present a summary of the results.

\section{Long-Baseline Experiments and Their Simulation}

Here we choose focusing on four experimental setups; two of them are already running: $\mathrm{T} 2 \mathrm{~K}$ [57] and $\mathrm{NO} \nu \mathrm{A}$ [58]; and two 
TABLE 1: Summary of neutrino experiments.

\begin{tabular}{lcccccc}
\hline Experiment & Baseline & Size & Target & Expected POT & Peak Energy $(\mathrm{GeV})$ & Status \\
\hline T2K [57] & $295 \mathrm{~km}$ & $22.5 \mathrm{kt}$ & Water & $7.8 \times 10^{21}\left(20 \times 10^{21}\right)$ & 0.6 & Running (10\% total POT) \\
NO $\nu$ A [58] & $810 \mathrm{~km}$ & $14 \mathrm{kt}$ & Liq. Scintillator & $3.6 \times 10^{21}$ & 2.0 & Running (17\% total POT) \\
DUNE [59] & $1300 \mathrm{~km}$ & $40 \mathrm{kt}$ & Liq. Argon & $1.47 \times 10^{21}$ & 2.5 & Start data taking: 2026 \\
T2HK [60] & $295 \mathrm{~km}$ & $2 \times 190 \mathrm{kt}$ & Water & $1.56 \times 10^{22}$ & 0.6 & Start data taking: 2026 (2032) \\
\hline
\end{tabular}

had their construction approved: DUNE [59] and T2HK [60]. Their sensitivity to the two most unknown parameters of the leptonic sector, the $\mathrm{CP}$ violation phase and the atmospheric mixing angle, makes them ideal to probe correlations among the mixing angles. As shown in [54], they can be used to shrink the parameter space of predictive models. A short description of each experiment can be found below and in Table 1.

(1) T2K. The Tokai to Kamiokande (T2K) experiment $[57,61]$ uses the Super-Kamiokande [62] as a far detector for the J-Park neutrino beam, which consists of an off-axis (by a $2.5^{\circ}$ angle) predominantly muon neutrino flux with energy around $0.6 \mathrm{GeV}$. The Super-Kamiokande detector is a $22.5 \mathrm{kt}$ water tank located at 295 from the J-Park facility. It detects neutrino through the Cherenkov radiation emitted by a charged particle created via neutrino interaction. There is also a near detector (ND280); thus the shape of the neutrino flux is well known, and the total normalization error reaches $5 \%$ for the signal and $10 \%$ for the background. T2K is already running and its current results can be found in [63] and reach $7 \times 10^{20} \mathrm{POT}$ of flux for each neutrino/antineutrino mode, which corresponds to $10 \%$ of the $7.8 \times 10^{21}$ expected approved exposure. There are also plans for extending the exposure to $20 \times 10^{21}$ POT.

(2) NOvA. The NuMI off-axis $v_{e}$ appearance $(\mathrm{NO} v \mathrm{~A})[58$, 64,65 ] is an off-axis (by a $0.8^{\circ}$ angle) that uses a neutrino beam from the Main Injector of Fermilab's beamline (NuMI). This beam consists of mostly muon neutrinos with energy around $2 \mathrm{GeV}$ traveling through $810 \mathrm{~km}$ until arriving at the 14 kt Liquid Scintillator far detector placed at Ash River, Minnesota. The far and near detectors are highly active tracking calorimeters segmented by hundreds of PVP cells and can give a good estimate of the total signal and background within an error of $5 \%$ and $10 \%$ of total normalization error, respectively. The planned exposure consists of a $3.6 \times 10^{21}$ POT that can be achieved in 6 years of running time, working in $50 \%$ in the neutrino mode and $50 \%$ in the antineutrino mode. $\mathrm{NO} v \mathrm{~A}$ is already running; current results can be found in $[66,67]$.

(3) DUNE. The Deep Underground Neutrino Experiment (DUNE) [59, 68-71] is a long-baseline next generation onaxis experiment also situated in Fermilab. It flux will be generated at the LBNF neutrino beam to target a $40 \mathrm{kt} \mathrm{Liquid}$ Argon time chamber projection (LarTPC) located $1300 \mathrm{~km}$ away from the neutrino source at Sanford Underground Research Facility (SURF). The beam consists of mostly muon neutrinos of energy around $2.5 \mathrm{GeV}$ and expects a total exposure of $1.47 \times 10^{21}$ POT running 3.5 years in neutrino mode and 3.5 years in antineutrino mode. The near and far detectors are projected to obtain a total signal (background) normalization uncertainty of $4 \%(10 \%)$. The experiment is expected to start taking data around 2026.

(4) T2HK. The Tokai to Hyper-Kamiokande (T2HK) [60, 7275] is an upgrade of the successful T2K experiment at J-Park. It uses the same beam as its predecessor $\mathrm{T} 2 \mathrm{~K}$, an off-axis beam from the J-Park facility $295 \mathrm{~km}$ away from its new far detector: two water Cherenkov tanks with $190 \mathrm{kt}$ of fiducial mass each. The expected total power is $1.56 \times 10^{22}$ POT to be delivered within $2.5 \mathrm{yrs}$ of neutrino mode and $7.5 \mathrm{yrs}$ of antineutrino mode in order to obtain a similar number of both neutrino types. The new design includes improvements in the detector systems and particle identification that are still in development. For simplicity, we take similar capability as the T2K experiment and will assume a 5\% (10\%) of signal (background) normalization error. The first data taking is expected to start with one tank in 2026 and the second tank in 2032.

In order to perform simulation of any neutrino experiment, the experimental collaboration uses Monte Carlo Methods, which can be performed through several event generators like GENIE [76], FLUKA [77], and many others. See PDG [78] for a review. Such technique requires an enormous computational power and detector knowledge, as it relies on the simulation of each individual neutrino interaction and how its products evolve inside of the detector. A simpler, but faster, simulation can be accomplished by using a semianalytic calculation of the event rate integral [79]:

$$
\begin{aligned}
& N_{i}\left(v_{\beta} \longrightarrow v_{\alpha}\right) \\
& \quad=\int_{E_{i}-\Delta E_{i} / 2}^{E_{i}+\Delta E_{i} / 2} K_{v_{\alpha}}\left(E, E^{\prime}\right) \phi_{\nu_{\beta}}(E) P_{\beta \alpha}(E) \sigma(E) d E d E^{\prime} .
\end{aligned}
$$

$N_{i}$ is the number of detected neutrinos with energy between $E_{i}-\Delta E_{i} / 2$ and $E_{i}+\Delta E_{i} / 2 . \phi_{\nu_{\beta}}(E)$ describes the flux of neutrino $\nu_{\beta}$ arriving at the detector. $P_{\beta \alpha}$ is the oscillation probability and $\sigma(E)$ the detection cross section of the detection reaction.

$K_{v_{\alpha}}\left(E, E^{\prime}\right)$, also known as migration matrix, describes how the detector interprets $\alpha$ neutrino with energy $E$ being detected at energy $E^{\prime}$ and summarizes the effect of the Monte Carlo simulation of the detector into a single function. A perfect neutrino detector is described by a delta function, 
TABLE 2: Description of the possible hypothesis taken to generate the numerical analysis. Here, Standard- $3 v$ means standard 3 neutrino oscillation.

\begin{tabular}{lccc}
\hline Cases & pseudo-data & Null Hypothesis & Test Hypothesis \\
General & $M_{i}$ & $M_{1}$ & $M_{2}$ \\
\hline I & Standard-3v & Standard-3v & New Model \\
II & New Model & Standard-3v & New Model \\
III & Standard-3v & New Model & Standard-3v \\
IV & New Model & New Model & Standard-3v \\
\hline
\end{tabular}

$K_{v_{\alpha}}\left(E, E^{\prime}\right)=\delta\left(E-E^{\prime}\right)$, while a more realistic simulation can use a Gaussian function:

$$
K_{v_{\alpha}}\left(E, E^{\prime}\right)=\frac{e^{-\left(E-E^{\prime}\right)^{2} / 2 \delta E^{2}}}{\sqrt{2 \pi} \delta E},
$$

where $\delta E$ parametrizes the error in the neutrino energy detection or a migration matrix provided by the experimental collaboration.

The public available software GLoBES [79, 80] follows this approach and is commonly used to perform numerical simulation of neutrino experiments. There is also another tool, the NuPro package [81] that will be publicly released soon. All the simulations in this manuscript are performed using GLoBES.

\section{Statistical Analysis and Probing Models: A Brief Discussion}

We are interested in a rule to distinguish between two neutrino oscillation models that can modify the spectrum of detected neutrinos in a long-baseline neutrino experiment. From the experimental point of view, one may apply a statistical analysis to quantitatively decide between two (or more) distinct hypotheses given a set of data points $H_{\text {real }}$.

Each model $\left(M_{i}\right)$ will define a probability distribution function (p.d.f.), $f\left(t \mid M_{i}\right)$, where the statistic test function $t$ depends on the real data points and the model parameters $\theta_{i}, i=1,2, \ldots$. The best fit of a model is defined as the values of the model parameters that maximize the p.d.f. function: $f_{0}\left(M_{i}\right)=\max \left[f\left(H_{\text {real }}, \theta_{i}^{1} \mid M_{i}\right)\right]$. Thus, one can reject model $M_{2}$, over model $M_{1}$ by some certain confidence level $n$ if

$$
\frac{f_{0}\left(M_{2}\right)}{f_{0}\left(M_{1}\right)} \leq C_{n} .
$$

$C_{n}$ is a constant that depends on the probability test, the number of parameters, and the confidence level $n$.

From the theoretical point of view, the real data points were not yet measured; this means that in order to find the expected experimental sensitivity we need to produce pseudo-data points $H_{\text {real }}$ by adding an extra assumption on which model is generating the yet-to-be-measured data points. That means there are various ways of obtaining sensitivity curves; each of them is described in Table 2.

Although one can always generate the pseudo-data points using any desired model at any point in its parameter space, the usual approach is to assume that the data points are
TABLE 3: Current best fit values of Standard-3v as given by [82]. Notice that Normal Hierarchy is assumed.

\begin{tabular}{lcc}
\hline parameter & value & error \\
\hline$\Delta m_{21}^{2} / 10^{-5}$ & $7.56 \mathrm{eV}^{2}$ & $(19)$ \\
$\Delta m_{31}^{2} / 10^{-3}$ & $2.55 \mathrm{eV}^{2}$ & $(4)$ \\
$\sin ^{2} \theta_{12}$ & 0.321 & $(18)$ \\
$\sin ^{2} \theta_{13}$ & 0.02155 & $(90)$ \\
$\sin ^{2} \theta_{23}$ & 0.430 & $(20)$ \\
$\delta_{\mathrm{CP}} / \pi$ & 1.40 & $(31)$ \\
\hline
\end{tabular}

generated by the standard 3 neutrino oscillation (Standard$3 v$ ) model with parameters given by current best fit values. We will use this approach in the work. Current best fit values are described in Table 3 and were taken from [82].

3.1. Frequentist Analysis. The chi-square test $[78,83,84]$ is the most common statistical analysis chosen to test the compatibility between the experimental data and the expected outcome of a given neutrino experiment. It is based on the construction of a Gaussian chi-squared estimator $\left(\chi^{2}\right)$ so that $f(t \mid$ Model $)=\mathrm{Ne}^{-\chi^{2} / 2}$. This means that the best fit values are obtained by the set of values that globally minimizes the function $\chi^{2}$. For long-baseline neutrino oscillation experiments the chi-square function can be divided into three factors:

$$
\chi^{2}=\chi_{\text {data }}^{2}+\chi_{\text {sys }}^{2}+\chi_{\text {prior }}^{2}
$$

where $\chi_{\text {data }}^{2}$ in the simplest case reduces to Poissonian Pearson's statistic

$$
\chi_{\text {data }}^{2}=\sum_{i}^{N}\left[\frac{N_{i}^{\mathrm{obs}}-(1-a) N_{i}^{s}-(1-b) N_{i}^{b}}{\sqrt{N_{i}^{\mathrm{obs}}}}\right]^{2} .
$$

$N_{i}^{\text {obs }}$ is the number of observed neutrinos in the bin $i=$ $1,2,3 \cdots N$. It represents the pseudo-data points generated by a given model. $N_{i}^{s}\left(N_{i}^{b}\right)$ is the signal (background) observed neutrino as expected by a given model and depends on the model parameters. $\chi_{\text {sys }}^{2}$ comprises the experimental uncertainties and systematics. For $\chi^{2}$ in (5), it is given by

$$
\chi_{\text {sys }}^{2}=\left(\frac{a}{\sigma_{a}}\right)^{2}+\left(\frac{b}{\sigma_{b}}\right)^{2} \text {. }
$$

Here, $\sigma_{a}\left(\sigma_{b}\right)$ is the total normalization error in the signal (background) flux. Finally, $\chi_{\text {prior }}^{2}$ contains all the prior information one wishes to include in the model parameters. In this work we will assume $\chi_{\text {prior }}^{2}=0$ unless stated otherwise.

The exponential nature of the chi-squared estimator makes it straightforward to find the confidence levels for the model parameters. It suffices to define the function

$$
\Delta \chi^{2}=\chi_{\min }^{2}\left(\theta_{i} \mid M_{2}\right)-\chi_{\min }^{2}\left(M_{1}\right),
$$

where $\chi_{\min }^{2}\left(M_{1}\right)$ is the chi-squared function assuming model $M_{1}$ calculated in its best fit and $\chi_{\min }^{2}\left(\theta_{i} \mid M_{2}\right)$ is the chisquared function assuming model $M_{2}$ minimized over all 
the desired free parameters. Thus, the confidence levels are obtained by finding the solutions of

$$
\Delta \chi^{2} \leq A_{n} .
$$

$\theta_{i}$ are all the fixed parameters of model $M_{2}$ and $A_{n}$ are the constants that define the probability cuts and depend on the number of parameters in $\chi^{2}\left(\theta_{i} \mid M_{2}\right)$ and the confidence probability. For $n \sigma$ intervals and one parameter, $A_{n}=n^{2}$.

Notice that $\Delta \chi^{2}$ is in fact a function of the parameters one assumes to generate the pseudo-data points, which we call True Values and denote as $\theta_{i}$ (True), and the parameters of the model we wish to test, which we call Test Values and denote as $\theta_{i}$ (Test).

\section{Measurement of Oscillation Parameters in Long-Baseline Experiments}

The main goal of long-baseline experiments is to measure with high precision the two most unknown oscillation parameters: the $\mathrm{CP}$ phase and the atmospheric mixing angle $\theta_{23}$ through the measurement of the neutrino/antineutrino $v_{\mu} \rightarrow v_{\mu}$ survival and $v_{\mu} \rightarrow v_{e}$ transition of neutrinos from the beamline. Many authors studied the power of longbaseline experiments to obtain the neutrino mixing parameters [85-93]. Particularly, only the transition is sensitive to $\delta_{\mathrm{CP}}$ and described, to first order in matter effects, by the probability function below.

$$
\begin{aligned}
& P\left(v_{\mu} \longrightarrow v_{e}\right)=4 c_{13}^{2} s_{13}^{2} s_{23}^{2} \sin ^{2} \Delta_{31} \\
& +8 c_{13}^{2} s_{12} s_{13} s_{23}\left(c_{12} c_{23} \cos \delta_{\mathrm{CP}}-s_{12} s_{13} s_{23}\right) \cos \Delta_{32} \\
& \cdot \cos \Delta_{31} \cos \Delta_{21}-8 c_{13}^{2} c_{12} c_{23} s_{12} s_{13} s_{23} \sin \delta_{\mathrm{CP}} \sin \Delta_{32} \\
& \cdot \sin \Delta_{31} \sin \Delta_{21}+4 s_{12}^{2} c_{13}^{2}\left(c_{12}^{2} c_{23}^{2}+s_{12}^{2} s_{23}^{2} s_{13}^{2}\right. \\
& \left.-2 c_{12} c_{23} s_{12} s_{23} s_{13} \cos \delta_{\mathrm{CP}}\right) \sin ^{2} \Delta_{21}-8 c_{13}^{2} s_{13}^{2} s_{23}^{2} \\
& \cdot \frac{a L}{4 E}\left(1-2 \sin _{13}^{2}\right) \cos \Delta_{32} \sin \Delta_{31}+8 c_{13}^{2} s_{13}^{2} s_{23}^{2} \\
& \cdot \frac{a}{\Delta m_{31}^{2}}\left(1-2 \sin _{13}^{2}\right) \sin ^{2} \Delta_{31},
\end{aligned}
$$

where $c_{i j}=\cos \theta_{i j}, s_{i j}=\sin \theta_{i j}, \Delta_{i j}=\Delta m_{i j}^{2} L / 4 E$, and $a=2 \sqrt{2} G_{\mathrm{F}} n_{e} E . G_{\mathrm{F}}$ is the Fermi constant and $n_{e}$ is the electron density in the medium. $E$ is the neutrino energy and $L$ is the baseline of the experiment and they are chosen to obey $L / E \sim 500$ in order to enhance the effect of the CP phase. The antineutrino probability is obtained by changing $a \rightarrow-a$ and $\delta_{\mathrm{CP}} \rightarrow-\delta_{\mathrm{CP}}$. Thus, the difference between neutrino and antineutrino comes from matter effects and the CP phase. It turns out that the T2HK is the most sensitivity to $\delta_{\mathrm{CP}}$ as it has a bigger statistic and lower matter effect and can reach $8 \sigma$ difference between $\mathrm{CP}$ conservation and maximal CPnonconservation [73], in contrast with DUNE's 5.5 $\sigma$ [68]. In Figure 1 we plotted the expected allowed regions of $\theta_{23}$ (test) versus $\delta_{\mathrm{CP}}$ (test) at $3 \sigma$ for each experiment. We assumed the true value of the parameters as those given in Table 3 . The

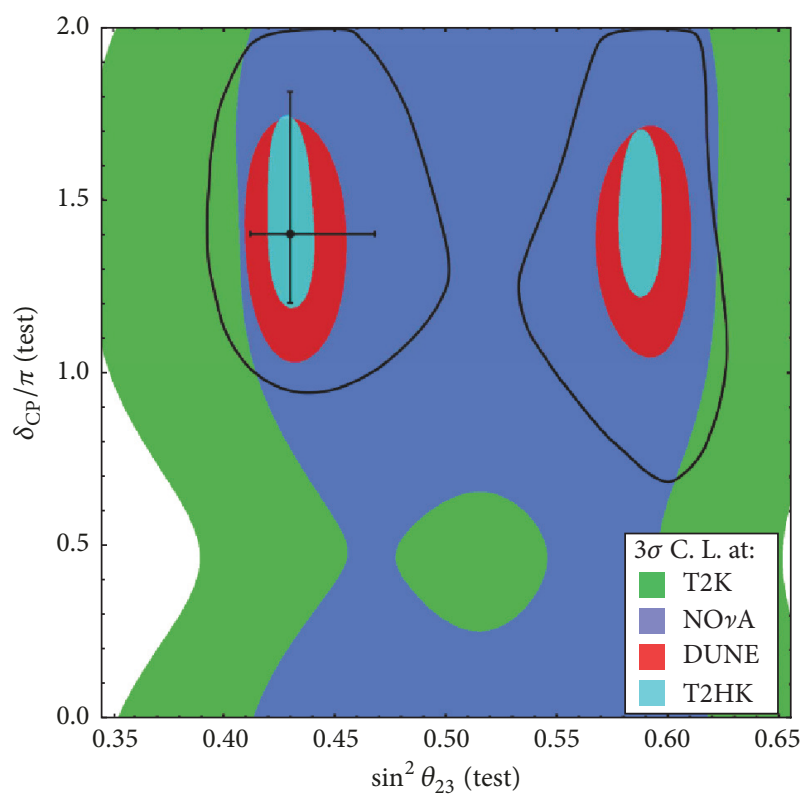

Figure 1: Expected sensitivity regions of $\sin ^{2} \theta_{23}$ (test) versus $\delta_{\mathrm{CP}}$ (test) assuming as the true and test model the Standard- $3 v$ paradigm for the three long-baseline detectors discussed: (1) T2K (green), (2) NO $v$ A (blue), (3) DUNE (red), and (4) T2HK (cyan). The black curve is current $90 \% \mathrm{CL}$ and the black point is the current best fit given in Table 3 . Notice that within this assumptions the octant would remain unresolved even at $3 \sigma \mathrm{CL}$.

black region is the current $90 \%$ CL region and the black points are the best fit points. T2HK is the most sensitive experiment in reconstructing both parameters, followed by DUNE. NO $v \mathrm{~A}$ and $\mathrm{T} 2 \mathrm{~K}$ are the first experiments to measure a difference between matter and antimatter in the leptonic sector but cannot measure the $\mathrm{CP}$ phase with more than $3 \sigma$. Notice that the experiments cannot discover the correct octant of $\theta_{23}$ at $3 \sigma$; that is, they cannot tell if $\theta_{23}>\pi / 4$ (High Octant) or $\theta_{23}<\pi / 4$ (Lower Octant) unless they are supplemented by an external prior. This effect is independent of the value of $\theta_{23}$ as can be observed in Figure 2(a) where we plotted the reconstruction of $\theta_{23}$ (test) given a fixed true value of $\theta_{23}$ (true) of each experiment. The black line corresponds to current best fit and the gray area is the $1 \sigma$ region. The $x$ like pattern of the region shows that given any true value of $\theta_{23}$ there is $3 \sigma$ region in the correct octant and in the wrong octant. Nevertheless, the octant can be obtained if one incorporates a prior to the $\theta_{13}$ angle [94-98] and future prospects on the measurement of $\theta_{13}$ by reactor experiments will allow both DUNE and T2HK to measure the octant if the atmospheric angle is not all inside the region $0.47<\sin ^{2} \theta_{23}<$ 0.53 [99].

For completeness, we show in Figure 2(a) the reconstruction of the $\delta_{\mathrm{CP}}$ (test) given a fixed true value of $\delta_{\mathrm{CP}}$ (true). The black line represents current best fit and the gray area shows the $1 \sigma$ region. We do not show the plots for $\mathrm{NO} \nu \mathrm{A}$ or $\mathrm{T} 2 \mathrm{~K}$ as they cannot reconstruct the $\mathrm{CP}$ phase at $3 \sigma$. The sensitivity is a little bit worse around maximum $\mathrm{CP}$ violation $\delta_{\mathrm{CP}}=\pi / 2$ or 


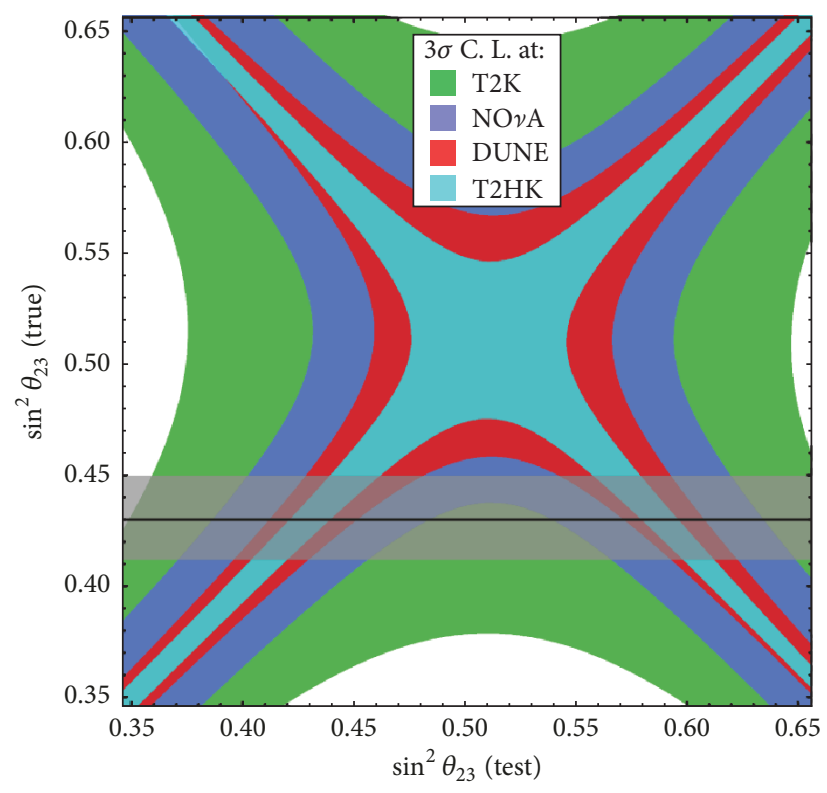

(a)

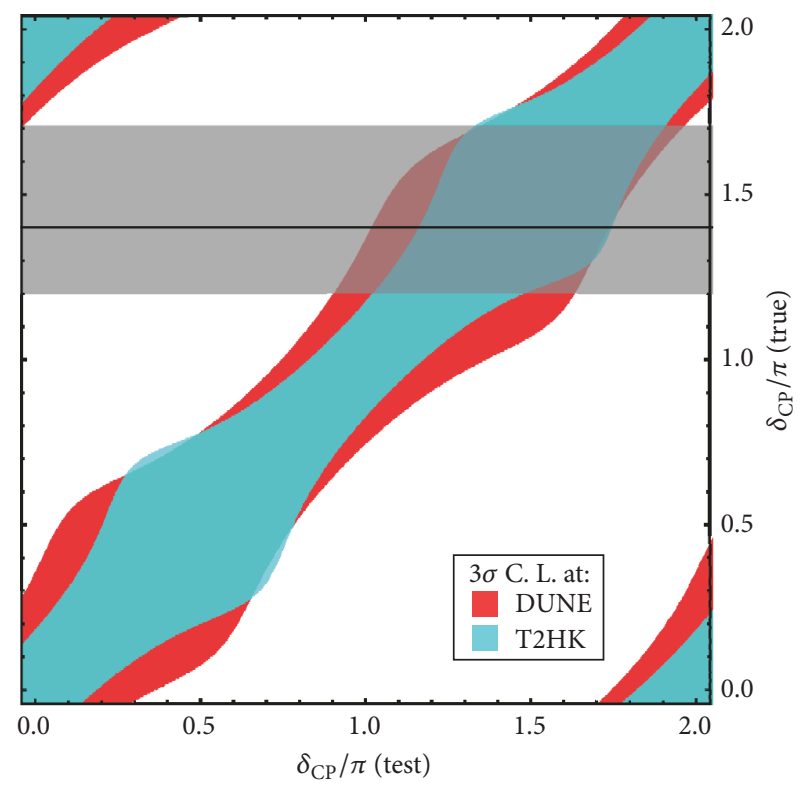

(b)

FIgURE 2: (a) and (b) correspond to the expected reconstruction of the oscillation parameter $\theta_{23}\left(\delta_{\mathrm{CP}}\right)$. The black line indicates the best fit value given in Table 3 and the gray area corresponds to its $1 \sigma$ region. The colored areas represent the regions that the experiments cannot distinguish within more than $3 \sigma$ for (1) T2K (green), (2) NOvA (blue), (3) DUNE (red), and (4) T2HK (cyan). In (b) we did not include T2K or $\mathrm{NO} v \mathrm{~A}$ as they cannot reconstruct the $\mathrm{CP}$ phase with more than $3 \sigma$.

$3 \pi / 2$ but in general it does not change much when one varies $\delta_{\mathrm{CP}}($ true $)$.

\section{5. $\theta_{23}$ and $\delta_{C P}$ Correlation and Probing Models}

In spite of being with relatively low energy $(<$ few $\mathrm{GeV})$, neutrino experiments can be a tool to probe high energy physics. Many neutrino mass models predict relations such as neutrino mass sum rules $[41,100-106]$ that can be probed in neutrinoless double beta decay [107] and relations among the neutrino mixing parameters. To name but a few examples we cite $[42-44,108]$. They can be put to test by a scan of the parameter space much like what was done by the LHC in search for new physics. Thus, inspired by the precision power of future long-baseline neutrino experiments, it was shown in [54] that models that predict a sharp correlation between the atmospheric angle and the CP phase can be used to put stringent bounds on parameters of such models.

In general, a predictive neutrino mass model $\mathscr{M}$ is constructed by imposing a symmetry on the Lagrangian and can be parametrized by a set of free parameters $\phi_{i}, i=$ $1,2, \ldots, N$, which can be translated into the usual neutrino mixing parameters from the neutrino mass matrix; that is,

$$
\begin{gathered}
\theta_{j k} \equiv \theta_{j k}\left(\phi_{i}\right), \\
\delta_{\mathrm{CP}} \equiv \delta_{\mathrm{CP}}\left(\phi_{i}\right) .
\end{gathered}
$$

Because of the symmetry on the Lagrangian, not all possible mass matrices are allowed to be generated and the free parameters $\phi_{i}$ may not span the entire space of the mixing parameters $\theta_{i j}$ and $\delta_{\mathrm{CP}}$. Thus, in principle, it is possible to probe or even exclude a model if the real best fit falls into a region that the model $\mathscr{M}$ cannot predict. As an example, in Figure 3 we plot the allowed parameter space of two discrete symmetry based models, the Warped Flavor Symmetry (WFS) model [45] (a) and the Revamped $A_{4}$ BabuMa-Valle (BMV) model [109] (b). The black curves represent currently unconstrained (Standard-3v) 90\% CL regions for the neutrino parameters and the black point shows the best fit value, while the blue region represents the $3 \sigma$ allowed parameter space of the two models. Notice that even for the $3 \sigma$ range the model can only accommodate a much smaller region than the unconstrained one. This is a reflex of the symmetries forced upon those models by construction; in WFS a maximal CP phase implies $\theta_{23}=\pi / 4$, and the smaller the $\mathrm{CP}$ violation is, the farther away from $\pi / 4$ the atmospheric angle is, while in BMV a maximal CP phase implies a Lower Octant atmospheric mixing and it cannot fit a $\left|\theta_{23}-\pi / 4\right|>$ $0.02 \pi$.

By using this approach, a full scan of the parameter space was performed for those two models, in [55] for the WFS model and in [56] for the Revamped $A_{4}$ model.

We show in Figure 4 an updated version of their results. The colored regions represent regions of the parameter space in which the model cannot be excluded with more than $3 \sigma$ for DUNE (red) and T2HK (cyan) experiment; both T2K and $\mathrm{NO} v \mathrm{~A}$ cannot probe the CP phase with more than $3 \sigma$; thus, they cannot exclude the model alone.

This means that if future long-baseline experiments measure a specific combination of $\delta_{\mathrm{CP}}$ and $\theta_{23}$ as its best fit that does not fall into the colored regions, they may be able 


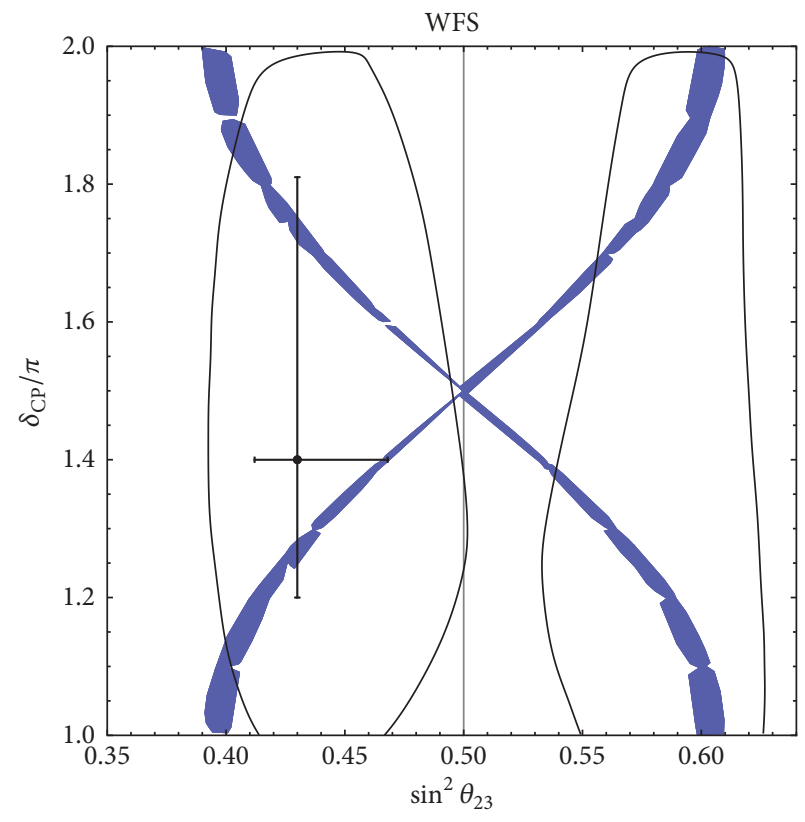

Model $3 \sigma$ C. L.

Uncons. 90\% C. L.

(a)

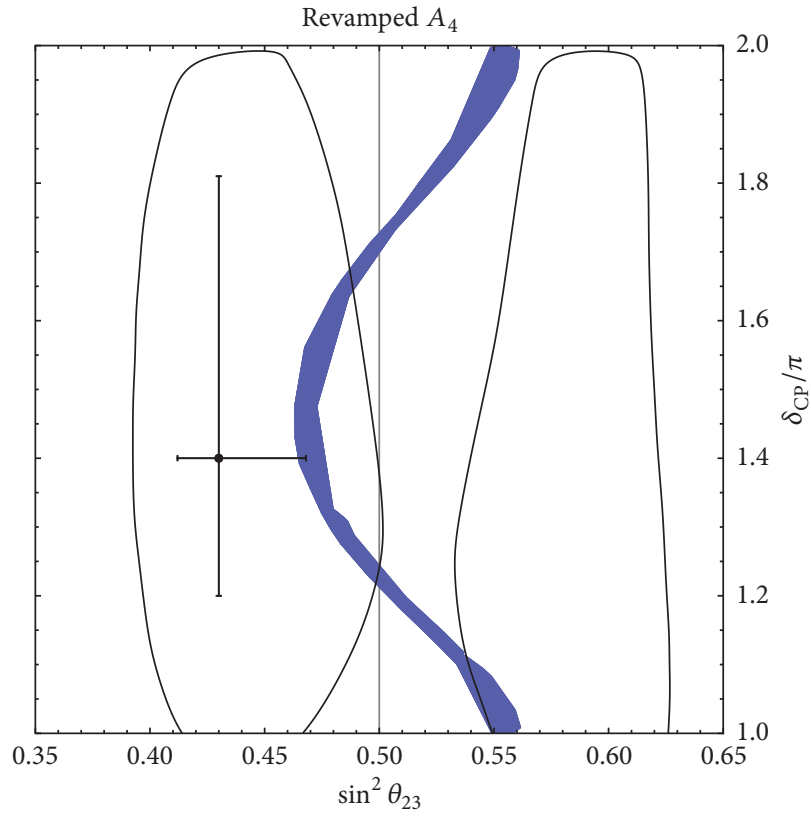

Model $3 \sigma$ C. L.

Uncons. 90\% C. L.

(b)

FIGURE 3: In blue are possible parameter values allowed by the two benchmark models: Warped Flavor Symmetry (a) and Revamped $A_{4}$-BMV (b). The regions are constructed by varying all the free parameters of the model and selecting those that are allowed at $3 \sigma$ in current global fit analysis [82]. The black line corresponds to current 90\% CL region and the black-dot is the best fit of Table 3. Normal Hierarchy is assumed.

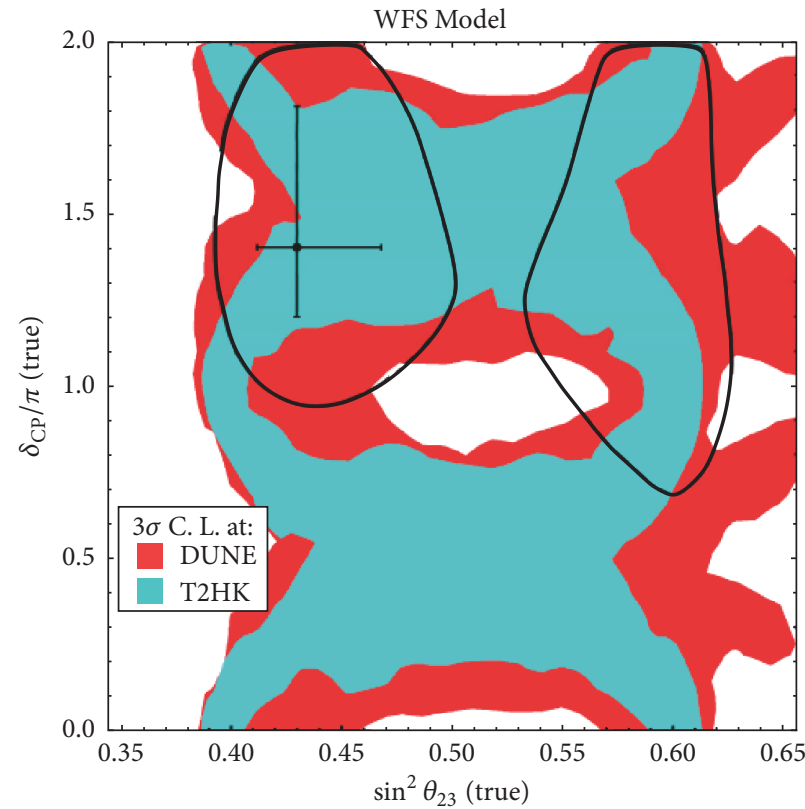

(a)

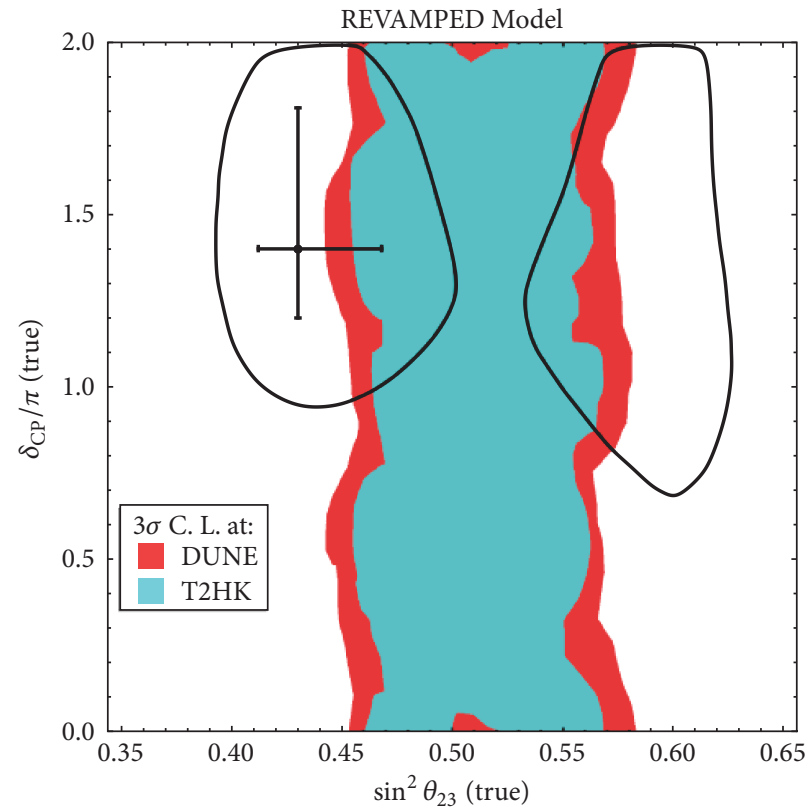

(b)

FIGURE 4: Expected sensitivity regions at which DUNE (Red) or T2HK (Cyan) would not exclude the WFS model (a) and the Revamped BMV model (b) at $3 \sigma$ confidence level. The black contours correspond to $90 \%$ CL of current global fit [82]. 
to exclude the model. Therefore, those kinds of analysis are guidelines to decide which model can or cannot be tested given the future results of DUNE and T2HK and are worth performing in any model that contains predictive correlations among the CP phase and the atmospheric mixing, like [42$44,110]$ and many others. It is also worth mentioning that combination of long-baseline measurements and reactors can greatly improve the sensitivity of the analysis.

\section{6. $\theta_{13}$ and the Atmospheric Octant}

The analysis in the last section can be extended to include another type of correlation that tries to explain the smallness of the reactor angle $\theta_{13} \sim O\left(10^{\circ}\right)$. A general approach common in many models [46-53] imposes a given symmetry on the mass matrix that predicts $\theta_{13}=0$, which is later spontaneously broken to give a small correction $\delta \theta_{13} \sim$ $O\left(10^{\circ}\right)$ to the reactor angle. It turns out that in order to generate nonzero $\theta_{13}$ one automatically generates corrections to other mixing angles $\delta \theta_{i j} \neq 0$.

This can be easily observed by considering a toy model that predicts the tri-bimaximal mixing matrix:

$$
U_{\mathrm{PMNS}}=U_{\mathrm{TBM}}=\left(\begin{array}{ccc}
\sqrt{\frac{2}{3}} & \sqrt{\frac{1}{3}} & 0 \\
\sqrt{\frac{1}{3}} & -\sqrt{\frac{1}{3}} & \sqrt{\frac{1}{2}} \\
-\sqrt{\frac{1}{3}} & \sqrt{\frac{1}{3}} & \sqrt{\frac{1}{2}}
\end{array}\right) .
$$

Any consistent small correction to the mixing matrix should maintain its unitary character. Particularly, we can set a correction in the 2-3 planes via the matrix

$$
\delta U_{23}=\left(\begin{array}{ccc}
1 & 0 & 0 \\
0 & 1 & \delta \theta \\
0 & -\delta \theta & 1
\end{array}\right)
$$

Notice that $\delta U_{23} \cdot \delta U_{23}^{\dagger}=1+O\left(\delta \theta^{2}\right)$. If we change the mixing matrix (notice that the correction $\delta U_{23} \cdot U_{\mathrm{TBM}}$ cannot produce a nonzero $\theta_{13}$ ) by $U_{\mathrm{PMNS}}=U_{\mathrm{TBM}} \rightarrow U_{\mathrm{TBM}} \cdot \delta U_{23}$ then $\theta_{13}=$ $(1 / \sqrt{3})\left|\pi / 4-\theta_{23}\right|$. The general case can be described by an initial mixing matrix $U_{\text {PMNS }}=U_{0}$ that is later corrected by a rotation matrix $U_{i j}$ :

$$
\begin{aligned}
& U_{\mathrm{PMNS}}=U_{0} \longrightarrow \\
& U_{\mathrm{PMNS}}=U_{i j} \cdot U_{0} \text { or } U_{0} \cdot U_{i j}
\end{aligned}
$$

All the possible combinations of corrections from tribimaximal, bimaximal, and democratic mixing were considered in [111]. Particularly, one can investigate a general correlation of $\theta_{13}$ to the nonmaximality of the atmospheric angle:

$$
\theta_{13}=F\left(\delta \theta_{23}\right)
$$

TABLE 4: Summary of models containing reactor and atmospheric angle correlation. All the possible combinations of corrections from tri-bimaximal, bimaximal, and democratic mixing were considered in [111].

\begin{tabular}{lcc}
\hline Model & $f$ & $\theta_{13}^{0}[\mathrm{rad}]$ \\
\hline$[53]$ & $\sqrt{2}$ & 0 \\
{$[50,51]$} & 0.35 & {$[0,0.35]$} \\
{$[49]$} & 0.1 or 10 & 0.62 \\
{$[52]$} & $1 / \theta_{0}$ & {$[-1,1]$} \\
$U_{13} \cdot U_{\mathrm{TBM}}$ & 6.3 & 0 \\
$U_{12} \cdot U_{\mathrm{TBM}}$ & 6.3 & 0 \\
$U_{\mathrm{TBM}} \cdot U_{23}$ & $1 / \sqrt{3}$ & 0 \\
$U_{\mathrm{BM}} \cdot U_{23} U_{13}$ & $1 / 2$ & 0 \\
$U_{\mathrm{TBM}} \cdot U_{23} U_{12}$ & 2 & 0.157 \\
$U_{\mathrm{TBM}} \cdot U_{23} U_{13}$ & $1 / \sqrt{2}$ & 0 \\
$U_{\mathrm{TBM}} \cdot U_{13} U_{12}$ & $2 / \sqrt{2}$ & 0 \\
$U_{\mathrm{BM}} \cdot U_{13} U_{12}$ & $\sqrt{3 / 2}$ & 0 \\
$U_{\mathrm{BM}} \cdot U_{23} U_{12}$ & $\sqrt{3 / 2}$ & 0 \\
$U_{\mathrm{BM}} \cdot U_{23} U_{13}$ & $1 / 2$ & 0 \\
\hline
\end{tabular}

where $F$ is a function of the correction $\delta \theta_{23}=\left|\pi / 4-\theta_{23}\right|$. Long-baseline experiments alone are not too sensitive to changes in the reactor angle; nevertheless, it was shown in [112] that it is possible to use such correlation to probe the parameter space of such models by combining long-baseline and reactor experiments.

This can be accomplished in a model-independent approach by series expanding (14):

$$
\theta_{13}=f(0)+f^{\prime}(0)\left|\frac{\pi}{4}-\theta_{23}\right| \equiv \theta_{13}^{0}+f\left|\frac{\pi}{4}-\theta_{23}\right| .
$$

This encompasses both the uncorrelated (Standard-3v) case if one sets $f=0$ and assumes $\theta_{13}^{0}$ as a free parameter and the small correction case by setting $\theta_{13}^{0}=0$ and $f \neq 0$. In Table 4 we present many models that contain this kind of correlation and their possible parameters values for $f$ and $\theta_{13}^{0}$.

In Figure 6 we update the potential exclusion regions where models of the form $\theta_{13}^{0}=0$ can be excluded for each value of $\sin ^{2} \theta_{23}$ (true) at $3 \sigma$ by DUNE + reactors and T2HK + reactors. The true value of $\theta_{13}$ is set to the central value of Table 3 and its error is assumed to be $3 \%$. The colored regions represent the regions that cannot be excluded with more than $3 \sigma$. There we can see that models that contain strong correlations $(f>1.9)$ or weak correlations $(f<0.8)$ can be excluded from any set of atmospheric angles.

The general case for any $\theta_{13}^{0}$ is presented in Figure 6(a) for DUNE and for T2HK in Figure 6(b) for three values of $\sin ^{2} \theta_{23}: 0.43$ (green), 0.5 (cyan), and 0.6 (red). The region shrinks greatly as the true value of the atmospheric angle goes away from the maximal mixing $\theta_{23}$ (true) $=\pi / 4$. 


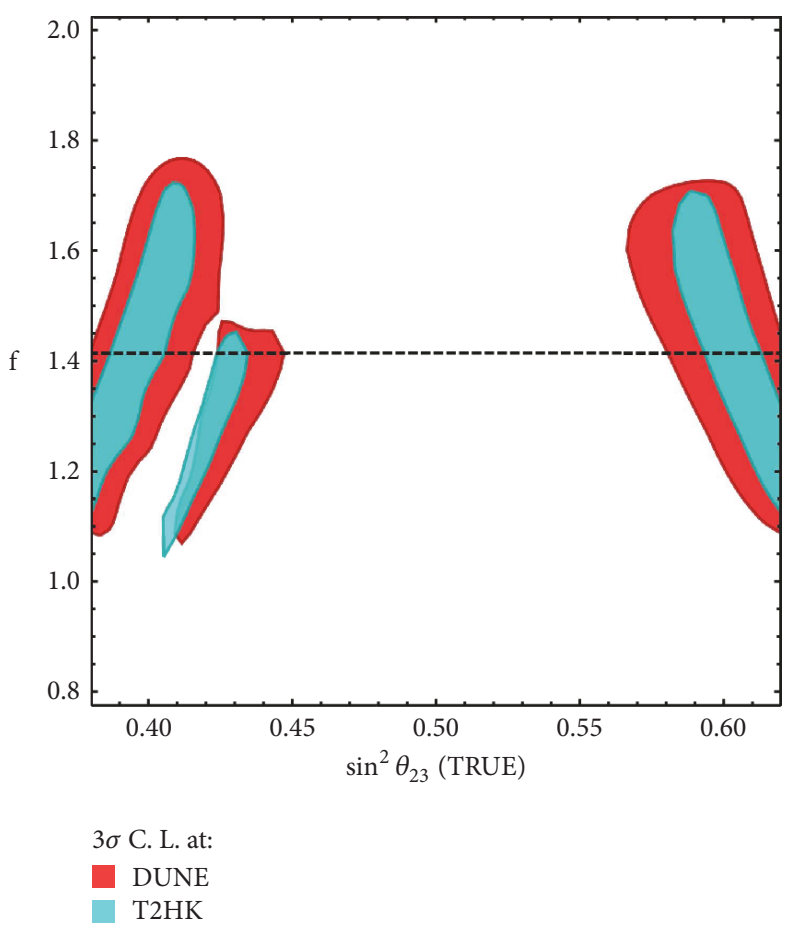

FIGURE 5: Regions that future neutrino long-baseline oscillation experiments cannot exclude the models that follow (15) at more than $3 \sigma \mathrm{CL}$ as a function of the true value of the atmospheric mixing angle for DUNE (red) and T2HK (cyan).

\section{Going Beyond Flavor Models}

Albeit flavor symmetry models are very common in the literature, mixing angles correlations are by no means exclusive to this class. Since long-baseline experiments are sensitive to the most unknown leptonic parameters, the possibility of using such correlations was studied not only in long-baseline but also in any neutrino experiment. The most common class is high energy model containing Nonstandard Interactions [114]; in particular, any model that produces nonstandard 4point Fermi interaction between electron and the neutrinos can, in principle, be probed by experiments that contain matter interactions, as well as studies in special mixing matrix ansatz such as Golden Ratio and other symmetries [41, 47, 91, 111, 113-118]. Moreover, one can find assumptions on neutrino mass sum rules that can be tested [104-106, 119] and generalized CP symmetry schemes [120-125]. General class of models such as grand unifying theories (GUT) and large extra dimensions (LED) was studied in [126-128]. Cosmology can also present ways of testing predictive neutrino mass models in leptogenesis [129] and even baryogenesis [130].

\section{Summary}

The state of the art of long-baseline neutrino oscillation experiments is $\mathrm{T} 2(\mathrm{H}) \mathrm{K}, \mathrm{NO} v$, and DUNE. They will be capable of reaching very good precision in the reactor and atmospheric mixing angle and will measure for the first time the CP violation phase. This will create an opportunity to put at test a plethora of neutrino mass models that predict values and correlations among the parameters of the PMNS matrix [54-56, 110, 131, 132].

Here we briefly discuss the fitting approach that quantifies the ability of long-baseline experiments to exclude predictive high energy models. Two types of correlations can be used: The $\theta_{23}-\delta_{\mathrm{CP}}$ correlation is found in many models containing a variety of symmetries [42-45]. Nevertheless, each model in the market may contain a different correlation, and most models are still in need to be analyzed. On the other hand, the $\theta_{13}-\theta_{23}$ correlation can only be probed by combining long-baseline with reactor experiments, as the former are not sensible enough to $\theta_{13}$ variations. However, we can take a model-independent approach [112] that covers most models that try to explain the smallness of the $\theta_{13}$ angle trough an spontaneous symmetry breaking [46-53]. We present a set of Figures 4, 5, and 6 containing the potential exclusion regions of each model here analyzed that can be used as a benchmark when the future experiments start to run.

\section{Conflicts of Interest}

The author declares that there are no conflicts of interest regarding the publication of this article. 


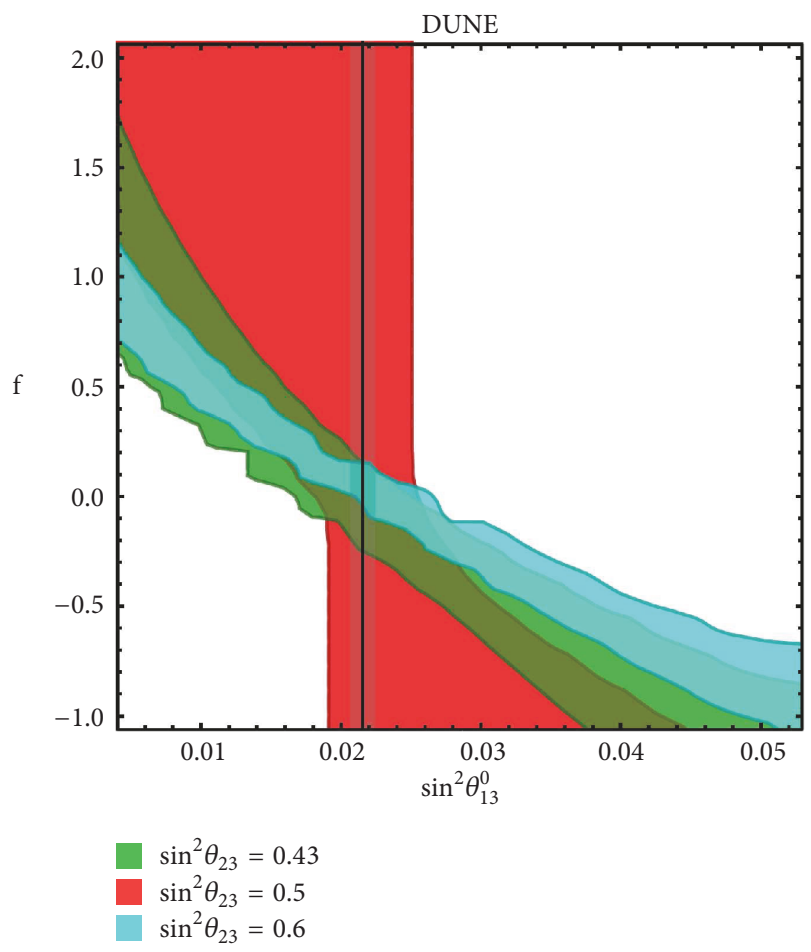

(a)

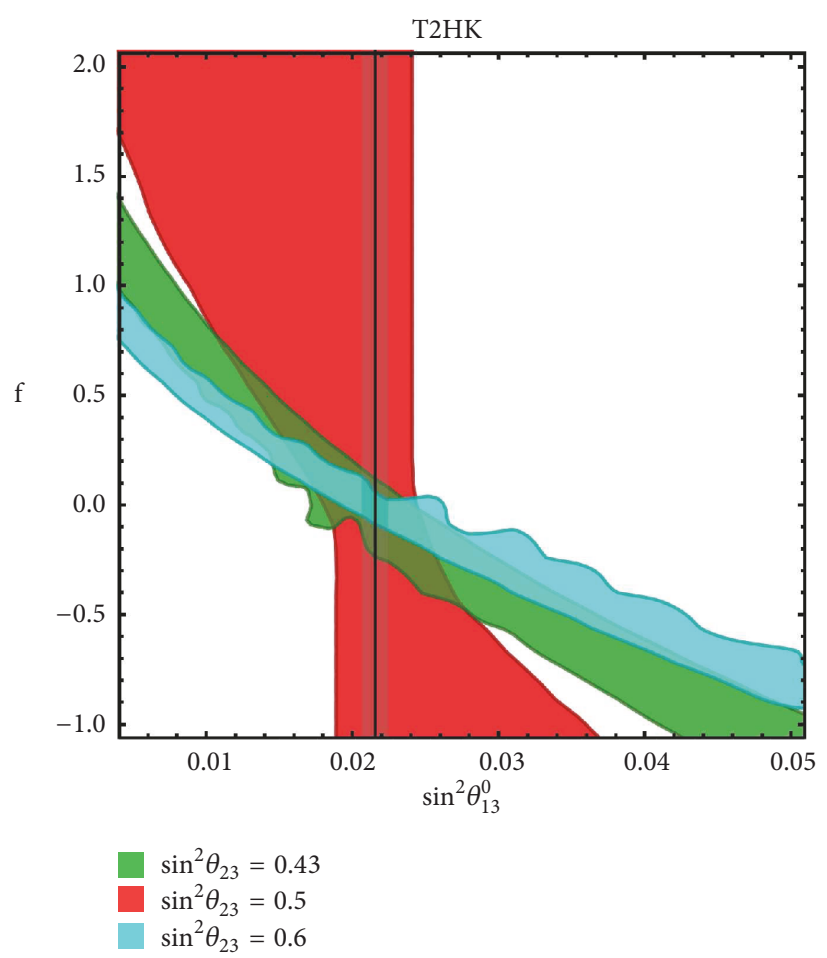

(b)

FIGURE 6: General parameter space regions that cannot be distinguished from the unconstrained relation hypothesis at more than $3 \sigma$ by future long-baseline neutrino oscillation experiments in combination with reactor measurements as a function of the two parameters of (15): $\theta_{13}^{0}$ and $f$. The analysis assumed the central value of the reactor angle as the best fit given in Table 3 and three values for the atmospheric angle: (1) $\sin ^{2} \theta_{23}$ (true) $=0.43$ (green), (2) $\sin ^{2} \theta_{23}$ (true) $=0.5$ (red), and (3) $\sin ^{2} \theta_{23}$ (true) $=0.6$ (cyan) for DUNE (a) and T2HK (b).

\section{Acknowledgments}

Pedro Pasquini was supported by FAPESP Grants 2014/051331, 2015/16809-9, and 2014/19164-6 and FAEPEX Grant no. 2391/17 and, also, by the APS-SBF collaboration scholarship.

\section{References}

[1] M. M. Guzzo, A. Masiero, and S. T. Petcov, "On the MSW effect with massless neutrinos and no mixing in the vacuum," Physics Letters B, vol. 260, no. 1-2, pp. 154-160, 1991.

[2] A. Bolanos, O. G. Miranda, A. Palazzo, M. A. Tortola, and J. W. F. Valle, "Probing nonstandard neutrino-electron interactions with solar and reactor neutrinos," Physical Review D: Particles, Fields, Gravitation and Cosmology, vol. 79, no. 11, Article ID 113012, 2009.

[3] Y. Farzan and M. Tortola, "Neutrino oscillations and NonStandard Interactions," 2017, https://arxiv.org/abs/1710.09360.

[4] M. Ghosh and O. Yasuda, "Testing NSI suggested by the solar neutrino tension in T2HKK and DUNE," 2017, https://arxiv.org/ abs/1709.08264.

[5] J. Tang and Y. Zhang, "Study of non-standard chargedcurrent interactions at the MOMENT experiment," 2017, https://arxiv.org/abs/1705.09500.

[6] J. Liao, D. Marfatia, and K. Whisnant, "Nonstandard neutrino interactions at DUNE, T2HK and T2HKK," Journal of High Energy Physics, vol. 71, 2017.
[7] Y. Farzan, "Viable models for large non-standard neutrino interactions," in Proceedings of the 18th International Workshop on Neutrino Factories, Superbeams, Beta beams (NuFact 2016), 7 pages, Quy Nhon, Vietnam, 2016, https://arxiv.org/abs/1612 .04971 .

[8] M. Blennow, P. Coloma, E. Fernandez-Martinez, J. HernandezGarcia, and J. Lopez-Pavon, "Non-unitarity, sterile neutrinos, and non-standard neutrino interactions," Journal of High Energy Physics, vol. 2017, no. 4, 2017.

[9] D. V. Forero and W. C. Huang, "Sizable NSI from the SU(2) scalar doublet-singlet mixing and the implications in DUNE," Journal of High Energy Physics, vol. 2017, article 018, no. 3, 2017.

[10] S. F. Ge and A. Y. Smirnov, "Non-standard interactions and the CP phase measurements in neutrino oscillations at low energies," Journal of High Energy Physics, vol. 2016, no. 10, 2016.

[11] M. Masud and P. Mehta, "Nonstandard interactions and resolving the ordering of neutrino masses at DUNE and other long baseline experiments," Physical Review D: Particles, Fields, Gravitation, and Cosmology, vol. 94, no. 5, Article ID 053007, 2016.

[12] P. Coloma and T. Schwetz, "Erratum: Generalized mass ordering degeneracy in neutrino oscillation experiments [Phys. Rev. D 94, 055005 (2016)]," Physical Review D: Particles, Fields, Gravitation, and Cosmology, vol. 95, no. 7, Article ID 079903, 2017.

[13] K. Huitu, T. J. Kärkkäinen, J. Maalampi, and S. Vihonen, "Constraining the nonstandard interaction parameters in long baseline neutrino experiments," Physical Review D: Particles, 
Fields, Gravitation, and Cosmology, vol. 93, no. 5, Article ID 053016, 2016.

[14] M. Blennow, S. Choubey, T. Ohlsson, D. Pramanik, and S. K. Raut, "A combined study of source, detector and matter non-standard neutrino interactions at DUNE," Journal of High Energy Physics, vol. 2016, no. 08, article no. 90, 2016.

[15] A. Boyarsky, D. Iakubovskyi, and O. Ruchayskiy, "Next decade of sterile neutrino studies," Physics of the Dark Universe, vol. 1, no. 1-2, pp. 136-154, 2012.

[16] K. M. Heeger, M. N. Tobin, B. R. Littlejohn, and H. P. Mumm, "Experimental parameters for a reactor antineutrino experiment at very short baselines," Physical Review D: Covering Particles, Fields, Gravitation, and Cosmology, vol. 87, no. 7, Article ID 073008, 2013.

[17] L. Gastaldo, C. Giunti, and E. Zavanin, "Light sterile neutrino sensitivity of $163 \mathrm{Ho}$ experiments," Journal of High Energy Physics, vol. 2016, no. 6, 2016.

[18] C. Giunti and E. M. Zavanin, "Appearance-disappearance relation in 3 + Ns short-baseline neutrino oscillations," Modern Physics Letters A, vol. 31, no. 01, p. 1650003, 2016.

[19] S. Gariazzo, C. Giunti, M. Laveder, Y. F. Li, and E. M. Zavanin, "Light sterile neutrinos," Journal of Physics G: Nuclear and Particle Physics, vol. 43, no. 3, Article ID 033001, 2016.

[20] O. Miranda, M. Tórtola, and J. Valle, "New Ambiguity in Probing," Physical Review Letters 6, 2016.

[21] D. Dutta and P. Ghoshal, "Probing CP violation with T2K, $\mathrm{NO} v \mathrm{~A}$ and DUNE in the presence of non-unitarity," Journal of High Energy Physics, vol. 2016, article 110, no. 9, 2016.

[22] D. Dutta, P. Ghoshal, and S. Roy, "Effect of non-unitarity on neutrino mass hierarchy determination at DUNE, $\mathrm{NO} v \mathrm{~A}$ and T2K," Nuclear Physics B, vol. 920, pp. 385-401, 2017.

[23] F. J. Escrihuela, D. V. Forero, O. G. Miranda, M. Tórtola, and J. W. F. Valle, "Probing CP violation with non-unitary mixing in long-baseline neutrino oscillation experiments: DUNE as a case study," New Journal of Physics, vol. 19, no. 9, Article ID 093005, 2017.

[24] S.-F. Ge, P. Pasquini, M. Tórtola, and J. W. F. Valle, "Measuring the leptonic $C P$ phase in neutrino oscillations with nonunitary mixing," Physical Review D: Particles, Fields, Gravitation, and Cosmology, vol. 95, no. 3, Article ID 033005, 2017.

[25] J. Hernandez-Garcia and J. Lopez-Pavon, "Non-Unitarity vs sterile neutrinos at DUNE," https://arxiv.org/abs/1705.01840.

[26] C. R. Das, J. Maalampi, J. Pulido, and S. Vihonen, "Determination of the $\theta_{23}$ octant in long baseline neutrino experiments within and beyond the standard model," 2017, https://arxiv.org/abs/1708.05182.

[27] C. Soumya and R. Mohanta, "Non-unitarity lepton mixing in an inverse seesaw and its impact on the physics potential of longbaseline experiments," High Energy Physics - Phenomenology, 24 pages, 2017.

[28] S. Choubey and D. Pramanik, "Constraints on sterile neutrino oscillations using DUNE near detector," Physics Letters B, vol. 764, pp. 135-141, 2017.

[29] M. Magg and C. Wetterich, "Neutrino mass problem and gauge hierarchy," Physics Letters B, vol. 94, no. 1, pp. 61-64, 1980.

[30] R. N. Mohapatra and G. Senjanovic, "Neutrino mass and spontaneous parity nonconservation," Physical Review Letters, vol. 44, p. 912, 1980.

[31] J. Schechter and J. W. Valle, "Neutrino-oscillation thought experiment," Physical Review D: Particles, Fields, Gravitation and Cosmology, vol. 23, no. 7, pp. 1666-1668, 1981.
[32] C. Wetterich, "Neutrino masses and the scale of $B-L$ violation," Nuclear Physics B, vol. 187, no. 2, pp. 343-375, 1981.

[33] R. Foot, H. Lew, X. G. He, and G. C. Joshi, "See-saw neutrino masses induced by a triplet of leptons," Zeitschrift für Physik C: Particles and Fields, vol. 44, no. 3, pp. 441-444, 1989.

[34] A. Abada, C. Biggio, F. Bonnet, M. B. Gavela, and T. Hambye, "Low energy effects of neutrino masses," Journal of High Energy Physics, vol. 2007, no. 12, article 061, 2007.

[35] F. Bonnet, M. Hirsch, T. Ota, and W. Winter, "Systematic study of the $d=5$ Weinberg operator at one-loop order," Journal of High Energy Physics, vol. 2012, no. 153, 2012.

[36] D. Aristizabal Sierra, A. Degee, L. Dorame, and M. Hirsch, "Systematic classification of two-loop realizations of the Weinberg operator," Journal of High Energy Physics, vol. 2015, no. 3, 2015.

[37] S. F. King, A. Merle, S. Morisi, Y. Shimizu, and M. Tanimoto, "Neutrino mass and mixing: from theory to experiment," New Journal of Physics, vol. 16, Article ID 045018, 2014.

[38] N. Haba, J. Sato, M. Tanimoto, and K. Yoshioka, "Possible flavor mixing structures of lepton mass matrices," Physical Review D: Particles, Fields, Gravitation and Cosmology, vol. 64, no. 11, 2001.

[39] P. Chen, G.-J. Ding, F. Gonzalez-Canales, and J. W. F. Valle, "Classifying CP transformations according to their texture zeros: Theory and implications," Physical Review D: Covering Particles, Fields, Gravitation, And Cosmology, vol. 94, no. 3, Article ID 033002, 2016, http://dx.doi.org/10.1103/PhysRevD.94 .033002 .

[40] G. Altarelli and F. Feruglio, "Discrete flavor symmetries and models of neutrino mixing," Reviews of Modern Physics, vol. 82, no. 3, pp. 2701-2729, 2010.

[41] S. F. King and C. Luhn, "Neutrino mass and mixing with discrete symmetry," Reports on Progress in Physics, vol. 76, no. 5, Article ID 056201, 2013.

[42] A. E. Cárcamo Hernández and H. N. Long, "A highly predictive $A_{4}$ flavour 3-3-1 model with radiative inverse seesaw mechanism," 2017, https://arxiv.org/abs/1705.05246.

[43] A. Dev, "Gauged $L_{\mu}-L_{\tau}$ Model with an Inverse Seesaw Mechanism for Neutrino Masses," 2017, https://arxiv.org/abs/1710 .02878 .

[44] S. Centelles Chuliá, R. Srivastava, and J. W. Valle, "Generalized bottom-tau unification, neutrino oscillations and dark matter: Predictions from a lepton quarticity flavor approach," Physics Letters B, vol. 773, pp. 26-33, 2017.

[45] P. Chen, G. Ding, A. D. Rojas, C. A. Vaquera-Araujo, and J. W. Valle, "Warped flavor symmetry predictions for neutrino physics," Journal of High Energy Physics, vol. 2016, no. 1, 2016.

[46] D. A. Dicus, S. F. Ge, and W. W. Repko, "Generalized hidden $\mathrm{Z}_{2}$ symmetry of neutrino mixing," Physical Review D: Particles, Fields, Gravitation and Cosmology, vol. 83, no. 9, Article ID 093007, 2011.

[47] M. Sruthilaya, C. Soumya, K. N. Deepthi, and R. Mohanta, "Predicting leptonic CP phase by considering deviations in charged lepton and neutrino sectors," New Journal of Physics, vol. 17, no. 8, Article ID 083028, 2015.

[48] A. Dev, P. Ramadevi, and S. U. Sankar, "Non-zero $\theta_{13}$ and $\delta_{C P}$ in a neutrino mass model with $\mathrm{A}_{4}$ symmetry," Journal of High Energy Physics, vol. 2015, article 034, no. 11, pp. 1-15, 2015.

[49] G. N. Li and X. G. He, "CP violation in neutrino mixing with $\delta$ $=-\pi / 2$ in $A_{4}$ Type-II seesaw model," Physics Letters B, vol. 750, pp. 620-626, 2015.

[50] D. N. Dinh, N. A. Ky, P. Q. Văn, and N. T. H. Vân, "A seesaw scenario of an $A_{4}$ flavour symmetric standard model," 2016, https://arxiv.org/abs/1602.07437. 
[51] N. A. Ky, P. Q. Văn, and N. T. H. Vân, "Neutrino mixing model based on an $A_{4} \times Z_{3} \times Z_{4}$ flavor symmetry," Physical Review D, vol. 94, no. 9, Article ID 095009, 2016.

[52] A. E. Cárcamo Hernández, S. Kovalenko, J. W. F. Valle, and C. A. Vaquera-Araujo, "Predictive Pati-Salam theory of fermion masses and mixing," Journal of High Energy Physics, vol. 2017, article 118, no. 7, 2017.

[53] P. H. Frampton, T. W. Kephart, and S. Matsuzaki, "Simplified renormalizable $T^{\prime}$ model for tribimaximal mixing and Cabibbo angle," Physical Review D: Particles, Fields, Gravitation and Cosmology, vol. 78, no. 7, Article ID 073004, 2008.

[54] P. Pasquini, S. C. Chuliá, and J. W. F. Valle, "Neutrino oscillations from warped flavor symmetry: Predictions for long baseline experiments T2K, NOvA, and DUNE," Physical Review D, vol. 95, no. 9, Article ID 095030, 2017.

[55] S. S. Chatterjee, P. Pasquini, and J. Valle, "Probing atmospheric mixing and leptonic $\mathrm{CP}$ violation in current and future long baseline oscillation experiments," Physics Letters B, vol. 771, pp. 524-531, 2017.

[56] S. S. Chatterjee, M. Masud, P. Pasquini, and J. Valle, "Cornering the revamped BMV model with neutrino oscillation data," Physics Letters B, vol. 774, pp. 179-182, 2017.

[57] K. Duffy et al., "Current Status and Future Plans of T2K," High Energy Physics - Experiment, 2017, https://arxiv.org/abs/1705 .01764 .

[58] S. Childress and J. Strait, "Long baseline neutrino beams at Fermilab," Journal of Physics: Conference Series, vol. 408, Article ID 012007, 2013.

[59] R. Acciarri, M. A. Acero, M. Adamowski et al., "Long-Baseline Neutrino Facility (LBNF) and Deep Underground Neutrino Experiment (DUNE) Conceptual Design Report Volume 2: The Physics Program for DUNE at LBNF," Instrumentation and Detectors, 2016, https://arxiv.org/abs/1512.06148.

[60] K. Abe, H. Aihara, C. Andreopoulos et al., "Physics potential of a long-baseline neutrino oscillation experiment using a J-PARC neutrino beam and Hyper-Kamiokande," Progress of Theoretical and Experimental Physics, vol. 2015, no. 5, Article ID 053C02, 2015.

[61] K. Abe, J. Adam, H. Aihara et al., "Neutrino oscillation physics potential of the T2K experiment," Progress of Theoretical and Experimental Physics, vol. 2015, no. 4, Article ID 043C01, 2015.

[62] K. Abe et al., "Solar neutrino measurements in SuperKamiokande-IV," Physical Review D: Particles, Fields, Gravitation, and Cosmology, vol. 94, no. 5, Article ID 052010, 2016.

[63] L. Haegel et al., "The latest T2K neutrino oscillation results," in Proceedings of the The European Physical Society Conference on High Energy Physics (EPS-HEP 2017), 6 pages, Venice, Italy, July 2017.

[64] R. B. Patterson, “The NOvA experiment: status and outlook," Nuclear Physics B - Proceedings Supplements, vol. 235-236, pp. 151-157, 2013.

[65] S. K. Agarwalla, S. Prakash, S. K. Raut, and S. U. Sankar, "Potential of optimized $\mathrm{NO} \nu \mathrm{A}$ for large $\theta_{13} \&$ combined performance with a LArTPC \& T2K," Journal of High Energy Physics, vol. 2012, Article ID 75, 2012.

[66] P. Adamson et al., "Constraints on Oscillation Parameters from $v_{e}$ Appearance and $\nu_{\mu}$ Disappearance in NOvA," Physical Review Letters, vol. 118, no. 23, Article ID 231801, 2017.

[67] P. Adamson et al., "Measurement of the Neutrino Mixing Angle $\theta_{23}$ in NOvA," Physical Review Letters, vol. 118, no. 15, Article ID 151802, 2017.
[68] R. Acciarri et al., "Long-Baseline Neutrino Facility (LBNF) and Deep Underground Neutrino Experiment (DUNE) Conceptual Design Report Volume 1: The LBNF and DUNE Projects," 2016, https://arxiv.org/abs/1601.05471.

[69] J. Strait et al., "Long-Baseline Neutrino Facility (LBNF) and Deep Underground Neutrino Experiment (DUNE) Conceptual Design Report Volume 3: Long-Baseline Neutrino Facility for DUNE June 24, 2015," https://arxiv.org/abs/1601.05823.

[70] R. Acciarri et al., "Long-Baseline Neutrino Facility (LBNF) and Deep Underground Neutrino Experiment (DUNE) Conceptual Design Report, Volume 4 The DUNE Detectors at LBNF," 2016, https://arxiv.org/abs/1601.02984.

[71] E. Kemp, “The Deep Underground Neutrino Experiment: The precision era of neutrino physics," Astronomische Nachrichten, vol. 338, no. 9-10, p. 993, 2017.

[72] K. Abe et al., "Letter of Intent: The Hyper-Kamiokande Experiment - Detector Design and Physics Potential -," 2011, https://arxiv.org/abs/1109.3262.

[73] Hyper-Kamiokande Collaboration, "Hyper-Kamiokande Design Report," KEK-PREPRINT-2016-21, ICRR-REPORT701-2016-1, 2016.

[74] K. Abe, Ke. Abe, H. Aihara et al., "The Hyper-Kamiokande Experiment," Tech. Rep., 2017, https://arxiv.org/abs/1705.00306.

[75] K. Abe, Ke. Abe, H. Aihara et al., "The Hyper-Kamiokande Experiment: Overview \& Status," Tech. Rep., 2017, https://arxiv .org/abs/1704.05933.

[76] C. Andreopoulos, A. Bell, D. Bhattacharya et al., "The GENIE neutrino Monte Carlo generator," Nuclear Instruments and Methods in Physics Research Section A: Accelerators, Spectrometers, Detectors and Associated Equipment, vol. 614, no. 1, pp. 87104, 2010.

[77] G. Battistoni, P. R. Sala, M. Lantz, A. Ferrari, and G. Smirnov, "Neutrino interactions: From theory to Monte Carlo simulations," in Proceedings of the 45th Karpacz Winter School in Theoretical Physics, vol. 40, p. 2491, Ladek-Zdroj, Poland, 2009.

[78] C. Patrignani, K. Agashe, G. Aielli et al., "Review of Particle Physics," Chinese Physics C, vol. 40, no. 10, Article ID 100001, 2016.

[79] P. Huber, M. Lindner, and W. Winter, "Simulation of longbaseline neutrino oscillation experiments with GLoBES: (General Long Baseline Experiment Simulator)," Computer Physics Communications, vol. 167, no. 3, pp. 195-202, 2005.

[80] P. Huber, J. Kopp, M. Lindner, M. Rolinec, and W. Winter, "New features in the simulation of neutrino oscillation experiments with GLoBES 3.0: (General Long Baseline Experiment Simulator)," Computer Physics Communications, vol. 177, no. 5, pp. 432-438, 2007.

[81] S.-F. Ge, "NuPro: a simulation package for neutrino properties," http://nupro.hepforge.org.

[82] P. F. de Salas, D. V. Forero, C. A. Ternes, M. Tortola, and J. W. F. Valle, "Status of neutrino oscillations 2017," 2017, https://arxiv.org/abs/1708.01186.

[83] F. James, Statistical Methods in Experimental Physics, 345 p, World Scientific, Hackensack, USA, 2006.

[84] W. G. Cochran, The Annals of Mathematical Statistics 23- No3, vol. 315, 1942.

[85] S. Prakash, S. K. Raut, and S. U. Sankar, "Getting the best out of T2K and NO vA," Physical Review D: Covering Particles, Fields, Gravitation, and Cosmology, vol. 86, no. 3, Article ID 033012, 2012. 
[86] M. Ghosh, P. Ghoshal, S. Goswami, and S. K. Raut, "Evidence for leptonic CP phase from NO $v$ A, T2K and ICAL: A chronological progression," Nuclear Physics B, vol. 884, pp. 274-304, 2014.

[87] M. Ghosh, S. Goswami, and S. K. Raut, "Maximizing the DUNE early physics output with current experiments," The European Physical Journal C, vol. 76, 114, 2016.

[88] M. Ghosh, P. Ghoshal, S. Goswami, N. Nath, and S. K. Raut, "New look at the degeneracies in the neutrino oscillation parameters, and their resolution by $\mathrm{T} 2 \mathrm{~K}, \mathrm{NO} v \mathrm{~A}$ and ICAL," Physical Review D: Covering Particles, Fields, Gravitation, and Cosmology, vol. 93, no. 1, Article ID 013013, 2016.

[89] K. Chakraborty, K. N. Deepthi, and S. Goswami, "Spotlighting the sensitivities of T2HK,T2HKK and DUNE," 2017, https://arxiv.org/abs/1711.11107.

[90] S. Choubey, D. Dutta, and D. Pramanik, "Measuring the Sterile Neutrino CP Phase at DUNE and T2HK," 2017, https://arxiv.org/ abs/1711.07464.

[91] S. K. Agarwalla, S. S. Chatterjee, S. T. Petcov, and A. V. Titov, "Addressing Neutrino Mixing Models with DUNE and T2HK," 2017, https://arxiv.org/abs/1711.02107.

[92] J. Evslin, S.-F. Ge, and K. Hagiwara, "The leptonic CP phase from $\mathrm{T} 2(\mathrm{H}) \mathrm{K}$ and $\mu^{+}$decay at rest," Journal of High Energy Physics, vol. 02, no. 137, 2016.

[93] P. Ballett, S. F. King, S. Pascoli, N. W. Prouse, and T. Wang, "Sensitivities and synergies of DUNE and T2HK," Physical Review D: Covering Particles, Fields, Gravitation, and Cosmology, vol. 96, no. 3, Article ID 033003, 2017.

[94] N. Nath, M. Ghosh, and S. Goswami, "The physics of antineutrinos in DUNE and determination of octant and $\delta_{C P}$," Nuclear Physics B, vol. 913, pp. 381-404, 2016.

[95] K. Bora, D. Dutta, and P. Ghoshal, "Determining the octant of $\theta_{23}$ at LBNE in conjunction with reactor experiments," Modern Physics Letters A, vol. 30, no. 14, Article ID 1550066, 22 pages, 2015.

[96] H. Minakata, H. Sugiyama, O. Yasuda, K. Inoue, and F. Suekane, "Erratum: Reactor measurement of $\theta_{13}$ and its complementarity to long-baseline experiments [Phys. Rev. D 68, 033017 (2003)]," Physical Review D: Particles, Fields, Gravitation and Cosmology, vol. 70, no. 5, Article ID 059901, 2004.

[97] S. K. Agarwalla, S. Prakash, and S. U. Sankar, "Resolving the octant of $\theta_{23}$ with $\mathrm{T} 2 \mathrm{~K}$ and $\mathrm{NO} v \mathrm{~A}$," Journal of High Energy Physics, vol. 07, 131, 2013.

[98] A. Chatterjee, P. Ghoshal, S. Goswami, and S. K. Raut, "Octant sensitivity for large $\theta_{13}$ in atmospheric and long-baseline neutrino experiments," Journal of High Energy Physics, vol. 06, 010, 2013.

[99] S. Sachi Chatterjee, P. Pasquini, and J. W. F. Valle, "Resolving the atmospheric octant by an improved measurement of the reactor angle," Physical Review D: Particles, Fields, Gravitation, and Cosmology, vol. 96, no. 1, Article ID 011303, 2017.

[100] S. F. King, "Parameterizing the lepton mixing matrix in terms of deviations from tri-bimaximal mixing," Physics Letters B, vol. 659, no. 1-2, pp. 244-251, 2008.

[101] D. Hernandez and A. Yu. Smirnov, "Lepton mixing and discrete symmetries," Physical Review D: Covering Particles, Fields, Gravitation, and Cosmology, vol. 86, no. 5, Article ID 053014, 2012.

[102] D. Hernandez and A. Yu. Smirnov, "Discrete symmetries and model-independent patterns of lepton mixing," Physical Review D: Covering Particles, Fields, Gravitation, and Cosmology, vol. 87, no. 5, Article ID 053005, 2013.
[103] P. Ballett, S. F. King, C. Luhn, S. Pascoli, and M. A. Schmidt, "Testing atmospheric mixing sum rules at precision neutrino facilities," Physical Review D: Particles, Fields, Gravitation and Cosmology, vol. 89, no. 1, Article ID 016016, 2014.

[104] M. Spinrath, "Neutrino Mass Sum Rules," Journal of Physics: Conference Series, vol. 888, no. 1, Article ID 012176, 2017.

[105] F. Buccella, M. Chianese, G. Mangano, G. Miele, S. Morisi, and P. Santorelli, "A neutrino mass-mixing sum rule from $\mathrm{SO}(10)$ and neutrinoless double beta decay," Journal of High Energy Physics, vol. 2017, no. 4, 2017.

[106] J. Gehrlein, A. Merle, and M. Spinrath, "Predictivity of neutrino mass sum rules," Physical Review D: Particles, Fields, Gravitation and Cosmology, vol. 94, no. 9, 2016.

[107] S. F. King, A. Merle, and A. J. Stuart, "The power of neutrino mass sum rules for neutrinoless double beta decay experiments," Journal of High Energy Physics, vol. 2013, no. 12, article no. $5,2013$.

[108] T. Wang and Y.-L. Zhou, "Neutrino non-standard interactions as a portal to test flavour symmetries," 2018, https://arxiv.org/ pdf/1801.05656.pdf.

[109] D. V. Forero, S. Morisi, J. C. Romão, and J. W. F. Valle, "Neutrino mixing with revamped $A_{4}$ flavor symmetry," Physical Review D: Particles, Fields, Gravitation, and Cosmology, vol. 88, no. 1, Article ID 016003, 2013.

[110] R. Srivastava, C. A. Ternes, M. Tórtola, and J. W. F. Valle, “Testing a lepton quarticity flavor theory of neutrino oscillations with the DUNE experiment," 2017, https://arxiv.org/abs/1711.10318.

[111] W. Chao and Y. j. Zheng, "Relatively large Theta13 from modification to the tri-bimaximal, bimaximal and democratic neutrino mixing matrices," Journal of High Energy Physics, vol. 2013, article 44, 2013.

[112] P. Pasquini, "Reactor and atmospheric neutrino mixing angles' correlation as a probe for new physics," Physical Review D: Particles, Fields, Gravitation, and Cosmology, vol. 96, no. 9, Article ID 095021, 2017.

[113] S. Pramanick, "Ameliorating the popular lepton mixings with A4 symmetry: A see-saw model for realistic neutrino masses and mixing," 2017, https://arxiv.org/abs/1711.03510.

[114] G.-J. Ding, S. F. King, and C.-C. Li, "Golden littlest seesaw," Nuclear Physics B, vol. 925, pp. 470-499, 2017.

[115] J. Zhang and S. Zhou, "Viability of exact tri-bimaximal, goldenratio and bimaximal mixing patterns and renormalizationgroup running effects," Journal of High Energy Physics, vol. 09, $167,2016$.

[116] L. A. Delgadillo, L. L. Everett, R. Ramos, and A. J. Stuart, "Predictions for the Dirac CP-Violating Phase from Sum Rules," 2018, https://arxiv.org/abs/1801.06377.

[117] C. H. Albright, A. Dueck, and W. Rodejohann, "Possible alternatives to tri-bimaximal mixing," The European Physical Journal C, vol. 70, no. 4, pp. 1099-1110, 2010.

[118] A. D. Hanlon, S.-F. Ge, and W. W. Repko, "Phenomenological consequences of residual $Z_{2}^{s}$ and $Z$ symmetries," Physics Letters $B$, vol. 729, pp. 185-191, 2014.

[119] J. Barry and W. Rodejohann, "Neutrino mass sum-rules in flavor symmetry models," Nuclear Physics B, vol. 842, no. 1, pp. 33-50, 2011.

[120] J. Turner, "Predictions for leptonic mixing angle correlations and nontrivial Dirac $C P$ violation from $A_{5}$ with generalized $C P$ symmetry," Physical Review D: Particles, Fields, Gravitation, and Cosmology, vol. 92, no. 11, Article ID 116007, 2015. 
[121] J. Turner, "Mixing angle and phase predictions from A5 with generalised CP," in Proceedings of the Topical Research Meeting on Prospects in Neutrino Physics (NuPhys'14), London, UK, 2015, https://arxiv.org/abs/1506.06898.

[122] P. Ballett, S. Pascoli, and J. Turner, "Mixing angle and phase correlations from $A_{5}$ with generalized $C P$ and their prospects for discovery," Physical Review D: Particles, Fields, Gravitation, and Cosmology, vol. 92, no. 9, Article ID 093008, 2015.

[123] L. L. Everett and A. J. Stuart, "Lepton sector phases and their roles in flavor and generalized CPsymmetries," Physical Review D: Particles, Fields, Gravitation, and Cosmology, vol. 96, no. 3, Article ID 035030, 2017.

[124] J.-N. Lu and G.-J. Ding, "Alternative schemes of predicting lepton mixing parameters from discrete flavor and CP symmetry," Physical Review D: Particles, Fields, Gravitation, and Cosmology, vol. 95, no. 1, Article ID 015012, 2017.

[125] P. Chen, S. Centelles Chuliá, G.-J. Ding, R. Srivastava, and J. W. F. Valle, "Neutrino Predictions from Generalized CP Symmetries of Charged Leptons," 2018, https://arxiv.org/abs/1802 .04275 .

[126] S. F. King, "Unified models of neutrinos, flavour and $C P$ Violation," Progress in Particle and Nuclear Physics, vol. 94, pp. 217-256, 2017.

[127] J. M. Berryman, A. de Gouvêa, K. J. Kelly, O. L. G. Peres, and Z. Tabrizi, "Large extra dimensions at the deep underground neutrino experiment," Physical Review D: Particles, Fields, Gravitation, and Cosmology, vol. 94, no. 3, Article ID 033006, 2016.

[128] M. Carena, Y.-Y. Li, C. S. Machado, P. A. N. Machado, and C. E. M. Wagner, "Neutrinos in large extra dimensions and shortbaseline $v_{e}$ appearance," Physical Review D: Particles, Fields, Gravitation, and Cosmology, vol. 96, no. 9, Article ID 095014, 2017.

[129] C.-C. Li and G.-J. Ding, "Implications of residual CP symmetry for leptogenesis in a model with two right-handed neutrinos," Physical Review D: Particles, Fields, Gravitation, and Cosmology, vol. 96, no. 7, Article ID 075005, 2017.

[130] H. Borgohain and M. K. Das, "Perturbations to $\mu-\tau$ symmetry, lepton Number Violation and baryogenesis in left-right symmetric Model," 2018, https://arxiv.org/abs/1803.05710.

[131] S. F. Ge, D. A. Dicus, and W. W. Repko, " $Z_{2}$ symmetry prediction for the leptonic Dirac CP phase," Physics Letters B, vol. 702, no. 4, pp. 220-223, 2011.

[132] S.-F. Ge, D. A. Dicus, and W. W. Repko, "Residual Symmetries for Neutrino Mixing with a Large theta_13 and Nearly Maximal delta_D," Physical Review Letters, vol. 108, Article ID 041801, 2012. 

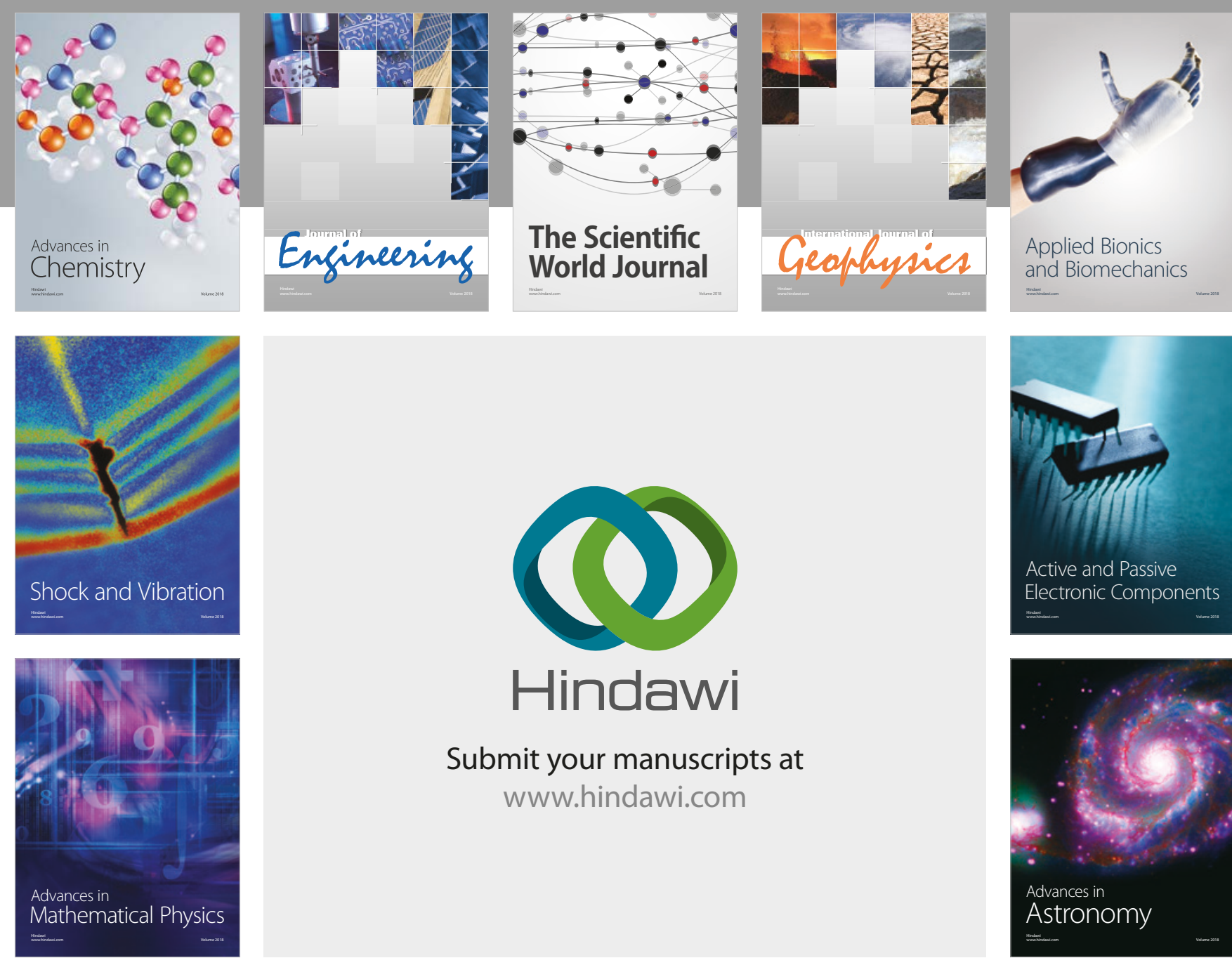

Submit your manuscripts at

www.hindawi.com

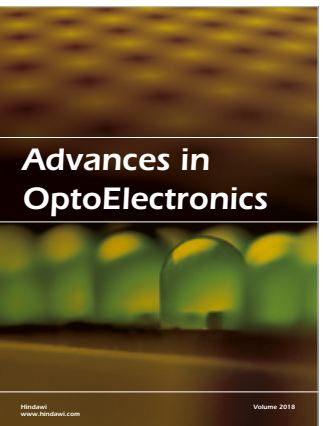

\section{Rotcting Machinery}
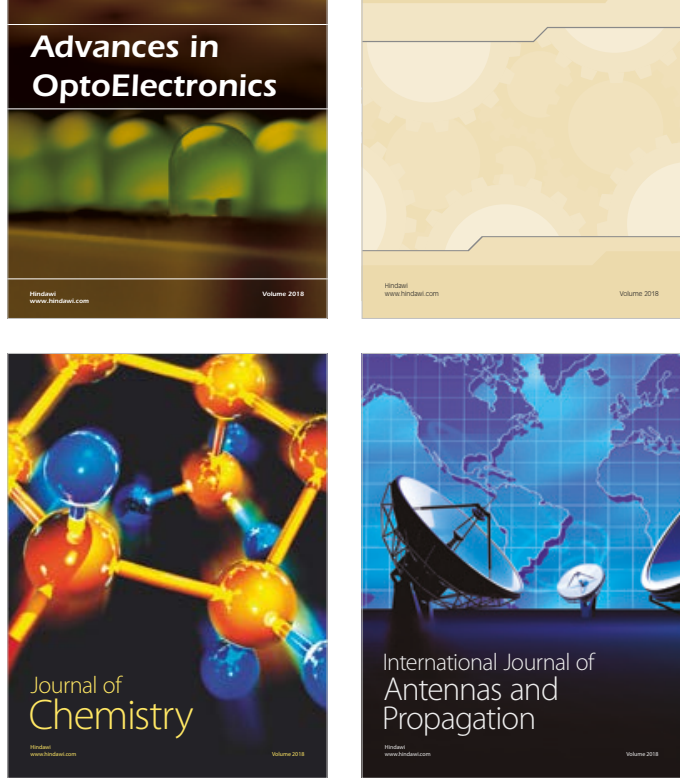

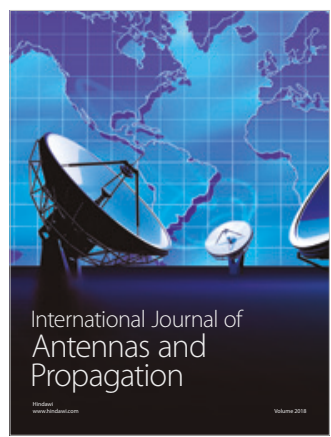

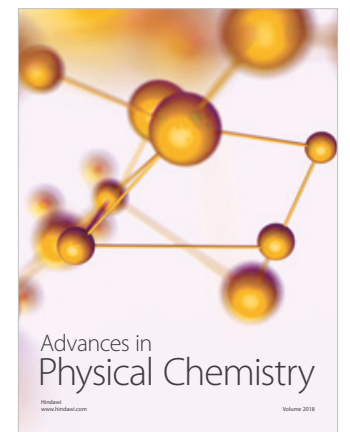

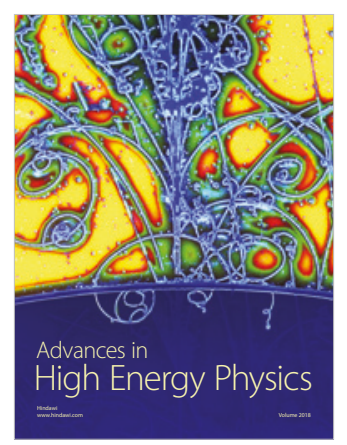

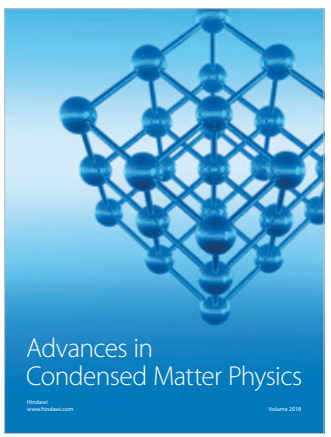

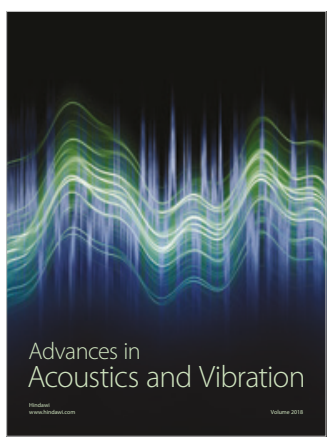

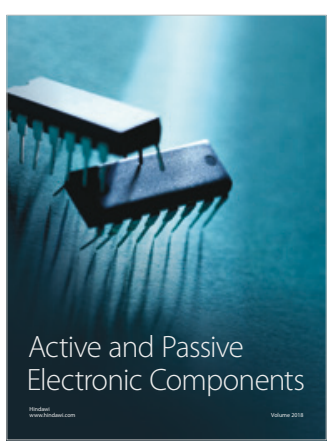
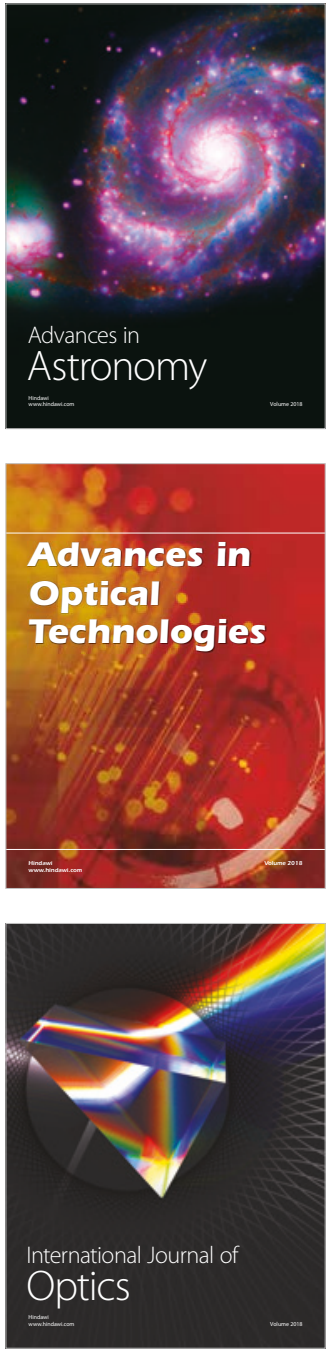